\section{EXTENSIVELY VALIDATED HLA LOH ALGORITHM DEMONSTRATES AN ASSOCIATION BETWEEN HLA LOH AND GENOMIC INSTABILITY}

${ }^{1}$ Rachel Pyke*, ${ }^{1}$ Steven Dea, ${ }^{1}$ Dattatreya Mellacheruvu, ${ }^{2}$ Charles Abbott, ${ }^{1}$ Simo Zhang, ${ }^{1}$ Lee McDaniel, ${ }^{1}$ Eric Levy, ${ }^{1}$ Gabor Bartha, ${ }^{1}$ John West, ${ }^{3}$ Michael Snyder, ${ }^{1}$ Richard Chen, ${ }^{1}$ Sean Boyle. ${ }^{1}$ Personalis, Oakland, USA; ${ }^{2}$ Pesronalis, Menlo Park, CA, USA; ${ }^{3}$ Stanford University, Menlo Park, USA

Background Human Leukocyte Antigen (HLA) genes are critical for the presentation of neoantigens to the immune system by cancer cells. Deletion of HLA alleles, known as HLA loss of heterozygosity (LOH), has been highlighted as a key immune escape mechanism. Validated algorithms to detect HLA LOH from sequencing data are critical for exploring the biological impact of HLA LOH and assessing its utility as a clinical biomarker.

Methods We developed DASH (Deletion of Allele-Specific HLAs), a machine learning algorithm trained on data from 279 patients on the ImmunoID NeXT Platform using features that account for probe capture variability between alleles and incorporate information from the regions flanking each HLA gene. To understand the contribution of boosted sequencing in the HLA region of the ImmunoID NeXT Platform, we performed an in silico downsampling analysis. To assess DASH's performance at variable tumor purities and HLA LOH subclonalities we identified three tumor-normal cell lines with HLA LOH and created in silico mixtures. Furthermore, after designing patient-specific primers for 21 patients that target specific alleles, we applied digital PCR (dPCR) to validate the HLA allele copy number status of the patients. Finally, we applied DASH to 611 patients spanning 15 tumor types.

Results In cross validation analyses across patient samples, DASH achieved $98.7 \%$ specificity and $92.9 \%$ sensitivity while LOHHLA, a widely used algorithm, only reached $94.3 \%$ and $78.8 \%$, respectively (figure 1). Downsampling analyses demonstrated that DASH benefits significantly from the boosted HLA sequencing on the ImmunoID NeXT Platform, dropping 0.06 in F-score after downsampling to the sequencing depth of other exome platforms. In cell line mixture analyses, DASH demonstrates greater than $99 \%$ specificity across all tumor purity and sub-clonality levels and greater than 98\% sensitivity for above $27 \%$ tumor purity. Moreover, DASH demonstrated $100 \%$ sensitivity and specificity in dPCR experiments across 21 tumor samples with stable controls. We applied DASH to a large pan-cancer cohort and found that $18 \%$ of patients had HLA LOH (figure 2). We identified strong associations between HLA LOH and genomic instability. Moreover, we demonstrated relationships between HLA LOH and markers of immune pressure, such as a correlation with CD274 (PD-1) expression and allele-specific neoantigen enrichment for deleted HLA alleles.

Conclusions DASH, a highly sensitive HLA LOH algorithm that has been extensively validated using cross validation, in silico downsampling, cell line mixtures and dPCR, has demonstrated the widespread impact of HLA LOH in a large pancancer cohort.

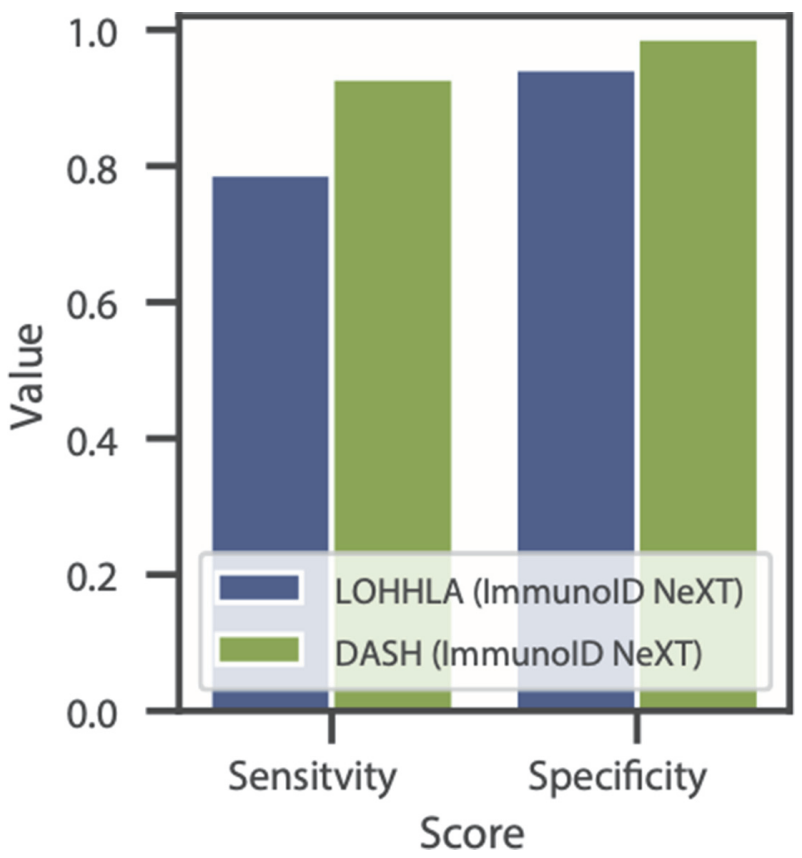

Abstract 79 Figure 1 Bar plots showing the sensitivity and specificities scores across ImmunoID NeXT cross validation samples between LOHHLA (blue) and DASH (green).
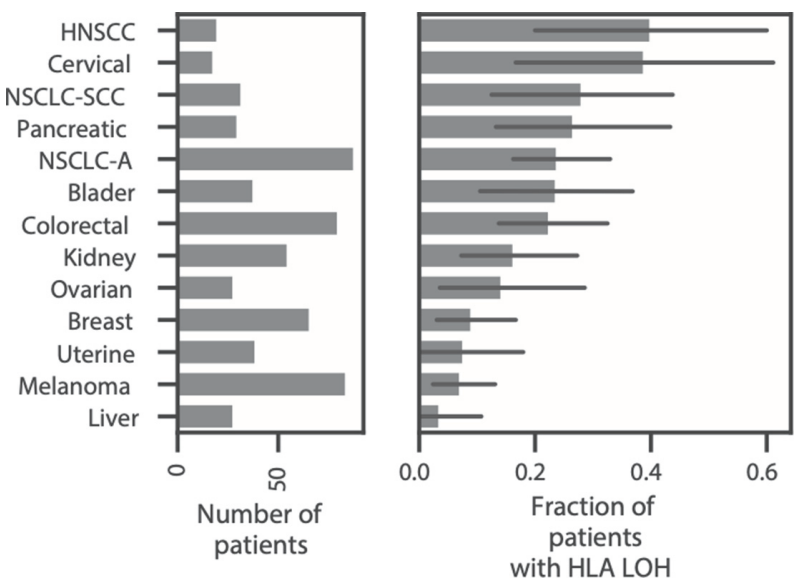

Abstract 79 Figure 2 Bar plots denoting the number of patients and the frequency of HLA LOH in each tumor type cohort. 95\% confidence intervals are shown with the thin dark grey bars. Only cohorts with at least 10 patients are shown

http://dx.doi.org/10.1136/jitc-2021-SITC2021.079 